\title{
Extracción y reciclaje de elementos nutritivos por cosecha de Eucalyptus globulus en Uruguay
}

\author{
Nutritious elements extraction and recycling at harvest of Eucalyptus globulus in Uruguay
}

\author{
Alejandro González a*, Jorge Hernández ${ }^{\text {b }}$, Amabelia del Pino ${ }^{\text {b }}$ \\ *Autor de correspondencia: ${ }^{a}$ UPM-Kymmene Corporation, Gerencia de Producción, CP 61.000, Tacuarembo, Uruguay, \\ tel./fax (+598)6324432, alejandro.gonzalez@upm.com \\ ${ }^{\text {b } U n i v e r s i d a d ~ d e ~ l a ~ R e p u ́ b l i c a, ~ F a c u l t a d ~ d e ~ A g r o n o m i ́ a, ~ D e p a r t a m e n t o ~ d e ~ S u e l o s ~ y ~ A g u a s, ~ M o n t e v i d e o, ~ U r u g u a y . ~}$
}

\begin{abstract}
SUMMARY
Eucalyptus commercial plantations extract high quantities of nutritious elements, although the proportion exported after harvest is relatively low. The aims of the work were: a) To quantify the concentration and distribution of nutritious elements in different components of a Eucalyptus globulus harvest b) To quantify decomposition rates of the harvest residues through the time, and the potential recycling of nutritious elements to the soil. In a 10 year-plantation on a Typic Hapludert, 24 trees with average height and DBH were harvested. The aerial biomass and total extraction of nitrogen, phosphorus, potassium, calcium and magnesium of logs, branches, bark and leaves were measured. For 24 months the decomposition of residues was measured with periodical samplings. The debarked commercial logs represented $77.5 \%$ of the produced biomass; and the proportions of nutritious elements exported with them were $32,45,19,18$ and $33 \%$ of nitrogen, phosphorus, potassium, calcium and magnesium respectively. The highest concentrations of nutritious elements were found in leaves and bark. The average residue decomposition was $30 \%$ of the initial aerial biomass. Leaves lost the highest proportion of biomass (68\%) and nutritious elements over 24 months, whereas the bark was the most resistant to decomposition. Potassium was released from the residues faster than the others, whereas calcium showed the lowest loss. Nitrogen, phosphorus and magnesium decreases were more gradual, proportional to the decomposition rates. De-barking in the site avoids the exportation of important amounts of nutritious elements.
\end{abstract}

Key words: decomposition rate, harvest residues, forest biomass.

\section{RESUMEN}

Las plantaciones comerciales de Eucalyptus globulus extraen altas cantidades de elementos nutritivos, aunque la proporción exportada luego de la cosecha es relativamente baja. Los objetivos del trabajo fueron: a) cuantificar la concentración y distribución de elementos nutritivos en diferentes componentes de cosecha de E. globulus; b) cuantificar las tasas de descomposición de residuos de cosecha en el tiempo, y el potencial reciclaje de elementos nutritivos al suelo. En una plantación de 10 años de edad sobre un Typic Hapludert se cosecharon 24 árboles con DAP y altura promedio. Se cuantificó la biomasa aérea y la extracción total de nitrógeno, fósforo, potasio, calcio y magnesio en trozas, ramas, corteza y hojas. Durante 24 meses se midió la descomposición de restos mediante muestreos periódicos. Las trozas descortezadas representaron $77,5 \%$ de la biomasa aérea, y las proporciones de elementos nutritivos exportadas con ellas fueron $32,45,19,18$ y $33 \%$ de nitrógeno, fósforo, potasio, calcio y magnesio, respectivamente. Las mayores concentraciones de elementos nutritivos se encontraron en corteza y hojas. La descomposición promedio de los restos fue $30 \%$ de la biomasa aérea inicial. Las hojas perdieron la mayor proporción de biomasa (68\%) y elementos nutritivos al término de 24 meses, mientras que la corteza fue más resistente a la descomposición. El potasio se liberó de los restos rápidamente, mientras que el calcio mostró la menor pérdida. Las disminuciones de nitrógeno, fósforo y magnesio fueron más graduales, proporcionales a las tasas de descomposición. El descortezado en el sitio evita la exportación de importantes cantidades de elementos nutritivos.

Palabras clave: tasa de descomposición, restos de cosecha, biomasa forestal.

\section{INTRODUCCIÓN}

Un sistema de producción forestal sostenible se basa en la aplicación de técnicas de manejo de suelos adecuadas, que tengan en cuenta aspectos productivos así como ambientales, tales como el control de la erosión, un balance adecuado de nutrientes, y la conservación del agua disponible del suelo. El balance de nutrientes, su reciclaje a lo largo del ciclo de producción, así como su conservación en los residuos de cosecha, resulta relevante para mantener el equilibrio del sistema en el largo plazo. Tanto la cuantificación del contenido de elementos nutritivos en las plantaciones forestales, así como la relación entre exportación/reciclaje y su disponibilidad para la reutilización 
posterior, resultan importantes para definir las estrategias de manejo sostenibles del ecosistema (Spangenberg et al. 1996). Entre las prácticas recomendadas, el mantener los residuos de la cosecha sobre la superficie del suelo no solo representa una reserva importante de nutrientes, sino que mejora las propiedades químicas, físicas y biológicas del mismo, obteniendo como resultado una mejora en la fertilidad del suelo (Smith et al. 1994, Gonçalves et al. 1997).

En Uruguay aún existe escasa información acerca de la extracción de elementos nutritivos por parte de diferentes bosques, así como en la posterior descomposición de los residuos de cosecha y reciclaje de dichos elementos al suelo. La velocidad de retorno al suelo y el grado de disponibilidad posterior para la reforestación es variable según la especie, el sitio, la edad de la plantación, el manejo de los restos, el nutriente en cuestión y su contenido en los restos, y la tasa de descomposición de cada componente de cosecha (Shammas et al. 2003, Palviainen et al. 2004). Hernández et al. (2009) encontraron que un alto porcentaje de los elementos nutritivos estaba presente en los residuos de cosecha de una plantación de Eucalyptus dunnii Maiden de 9 años, donde las trozas comerciales descortezadas exportaron menos del $40 \%$ de los elementos nutritivos estudiados (nitrógeno, fósforo, potasio, calcio y magnesio). La velocidad de descomposición de los restos de cosecha $\mathrm{y}$ el posterior reciclaje de los nutrientes al suelo es variable según su distribución sobre la superficie del suelo, el clima, así como también según la composición química de estos residuos. Herbert (1992) menciona importantes diferencias en el reciclaje de los elementos nutritivos luego de la cosecha del bosque, indicando para Eucalyptus nitens (Deane and Maiden) Maiden un $50 \%$ más de retorno de elementos nutritivos respecto a Eucalyptus grandis Hill ex Maiden. La relación carbono: nitrógeno de los restos, el contenido de nitrógeno, carbono soluble, lignina y polifenoles son determinantes en las tasas de descomposición de hojas, ramas y corteza (Rezende et al. 2001, Lovett et al. 2004, Verkaik et al. 2006).

Para Uruguay, si bien se ha estudiado cómo afectan dichas características a la tasa de descomposición de los residuos de cosecha para diferentes especies de Eucalyptus en condiciones controladas (Sánchez 2011), solo se cuenta con un estudio en condiciones de campo, y para una especie (E. dunnii), en el cual se evaluó la velocidad de descomposición de residuos durante dos años, y su incidencia en la liberación y disponibilidad de elementos nutritivos para la futura replantación (Hernández et al. 2009). Dada las características particulares del país, tanto climáticas (régimen pluviométrico, temperaturas) como edáficas, así como las propias de cada especie de Eucalyptus, es importante la generación de resultados locales.

Se plantea como hipótesis que el retorno de elementos nutritivos al suelo es variable en función del tiempo dependiendo del tipo de residuo involucrado y del elemento nutritivo en consideración, dadas las diferencias en su dinámica de reciclaje.
Los objetivos del presente trabajo son: a) cuantificar la concentración y distribución de nitrógeno, fósforo, potasio, calcio y magnesio en diferentes componentes de una cosecha de E. globulus; b) cuantificar las tasas de descomposición de los diferentes residuos de cosecha de E. globulus en función del tiempo, y el reciclaje potencial de nitrógeno, fósforo, potasio, calcio y magnesio al suelo.

\section{MÉTODOS}

Sitio experimental. El sitio experimental estuvo localizado en Palmitas (Departamento de Soriano, Uruguay, $57^{\circ} 48^{\prime} \mathrm{O}$ y $\left.33^{\circ} 25^{\prime} \mathrm{S}\right)$. La región presenta un clima templado, con temperatura media anual de $17,3{ }^{\circ} \mathrm{C}$, temperatura media de $10,9^{\circ} \mathrm{C}$ en el mes más frío (junio), temperatura media de $24,3{ }^{\circ} \mathrm{C}$ en el mes más cálido (enero), y precipitaciones anuales promedio de $1.130 \mathrm{~mm}$. El suelo del experimento es clasificado como fine, smectitic, thermic Typic Hapludert (Soil Survey Staff. 2010) (cuadro 1).

Evaluación de la biomasa aérea al momento de cosecha. El experimento fue realizado en un rodal de E. globulus de 10 años de edad, próximo a cosecha, y con destino a la producción de pasta de celulosa. El incremento medio anual (IMA) fue de $12,7 \mathrm{~m}^{3} \mathrm{ha}^{-1}$ año ${ }^{-1}$, considerado dentro del promedio de la especie para plantaciones de la década del 90 en el oeste uruguayo.

Se seleccionaron 24 árboles con medidas cercanas al promedio (DAP: diámetro a la altura del pecho, 1,30 m; y altura total), para lo cual se midieron todos los árboles existentes en una parcela de una hectárea (densidad: 970 árboles ha ${ }^{-1}$ ). Los árboles seleccionados tenían unos $20 \mathrm{~m}$ de altura y $20,5 \mathrm{~cm}$ de diámetro. Los mismos se apearon, separándose en cada uno de ellos los diferentes componentes: trozas comerciales de $2,4 \mathrm{~m}$ (diámetros $\geq 5 \mathrm{~cm}$ ) y residuos de cosecha (corteza, ramas y hojas). Se tomaron muestras de tres ramas de tamaño promedio para estimar en ellas las proporciones de ramas finas (diámetro $<1 \mathrm{~cm}$ ), medias (diámetros entre 1 y $2 \mathrm{~cm}$ ), gruesas (diámetro $>$ $2 \mathrm{~cm}$ ) y hojas. De esta manera se estimó la contribución proporcional de cada residuo a la biomasa total.

De cada troza se cortó un disco de $5 \mathrm{~cm}$ de espesor en la porción basal. De los restantes componentes de cosecha, se tomaron muestras compuestas por árbol. Todas las muestras fueron pesadas, secadas a estufa a $65^{\circ} \mathrm{C}$ hasta peso constante, calculando la tasa de descomposición durante el período en base al peso inicial y el remanente a la fecha de muestreo (Olson 1963).

Se calculó la biomasa aérea por unidad de superficie en base al peso promedio de los 24 ejemplares cosechados, y al número de árboles por hectárea.

Estudio de descomposición de restos de cosecha. Se realizó un estudio de descomposición de restos in situ, para lo cual se pesaron $100 \mathrm{~g}$ de los diferentes residuos de cosecha (ramas finas, ramas medias, corteza y hojas) colocándolos 
en bolsas individuales de tejido de malla (diámetro de 1 x $1 \mathrm{~mm}$ ) de fibra de $30 \times 30 \mathrm{~cm}$, y depositándolas sobre el mantillo forestal, simulando la situación post-cosecha. Se realizaron tres repeticiones de cada grupo de muestras.

Para cada residuo de cosecha fueron realizados muestreos a los $1,2,4,6,9,12,18$ y 24 meses post-cosecha, extrayendo una bolsa de cada componente (hojas, corteza, ramas finas y medias) por repetición. Por consiguiente, fueron ocho fechas, con cuatro muestras en cada fecha y tres repeticiones (total 96 muestras). En el laboratorio fueron secadas a estufa a $65^{\circ} \mathrm{C}$ hasta peso constante, calculando la tasa de descomposición durante el período en base al peso inicial y el remanente a la fecha de muestreo (Olson 1963).

Se tomaron registros de lluvias de una estación meteorológica cercana al experimento (figura 1). Durante el primer año de estudio (septiembre de 2005 a agosto de 2006) las precipitaciones fueron de $1.275 \mathrm{~mm}$, similares a la me- dia histórica para la zona, con dos picos de precipitaciones en los meses de enero y junio de 2006 ( 250 y $300 \mathrm{~mm}$, respectivamente); por su parte en el segundo año del estudio (septiembre de 2006 a agosto de 2007) las precipitaciones fueron casi el doble del promedio histórico de esa zona, con más de $2.000 \mathrm{~mm}$ en el período. El último cuatrimestre de 2006 registró $650 \mathrm{~mm}$ de lluvia, mientras que en los primeros ocho meses de 2007 llovieron casi $1.400 \mathrm{~mm}$, con un pico de $950 \mathrm{~mm}$ en el trimestre marzo-mayo. Esto indica que los niveles de humedad en el segundo año fueron muy superiores respecto a los del primero. Por su parte, la temperatura media y máxima promedio fueron superiores en el primer año de estudio respecto al segundo $\left(1,4\right.$ y $1,3{ }^{\circ} \mathrm{C}$, respectivamente). El invierno de 2007 fue excepcionalmente frío, registrándose 45 heladas agrometeorológicas. Se tomaron como referencias las medias históricas de precipitaciones para la zona (datos de la Dirección Nacional de Meteorología, 1960-2010).

Cuadro 1. Características del suelo estudiado.

Characteristics of the studied soil.

\begin{tabular}{|c|c|c|c|c|c|c|c|c|c|c|}
\hline \multirow{2}{*}{ Horizonte } & Profundidad & Arcilla & Arena & Limo & C orgánico & \multirow{2}{*}{$\mathrm{pH}\left(\mathrm{H}_{2} \mathrm{O}\right)$} & $\mathrm{Ca}$ & $\mathrm{Mg}$ & $\mathrm{K}$ & $\mathrm{Na}$ \\
\hline & $\mathrm{cm}$ & \multicolumn{3}{|c|}{-------- \% en peso------- } & $\mathrm{g} \mathrm{kg}^{-1}$ & & \multicolumn{4}{|c|}{------ cmol+ kg-1 ------ } \\
\hline A & $0-20$ & 33 & 39 & 28 & 21,2 & 5,2 & 11,50 & 2,45 & 0,30 & 0,30 \\
\hline $\mathrm{Bt}$ & $20-45$ & 45 & 24 & 31 & 17,5 & 5,8 & 15,60 & 2,84 & 0,31 & 0,39 \\
\hline $\mathrm{C}$ & $45+$ & 46 & 30 & 24 & 9,9 & 6,4 & 20,60 & 4,14 & 0,46 & 0,40 \\
\hline
\end{tabular}

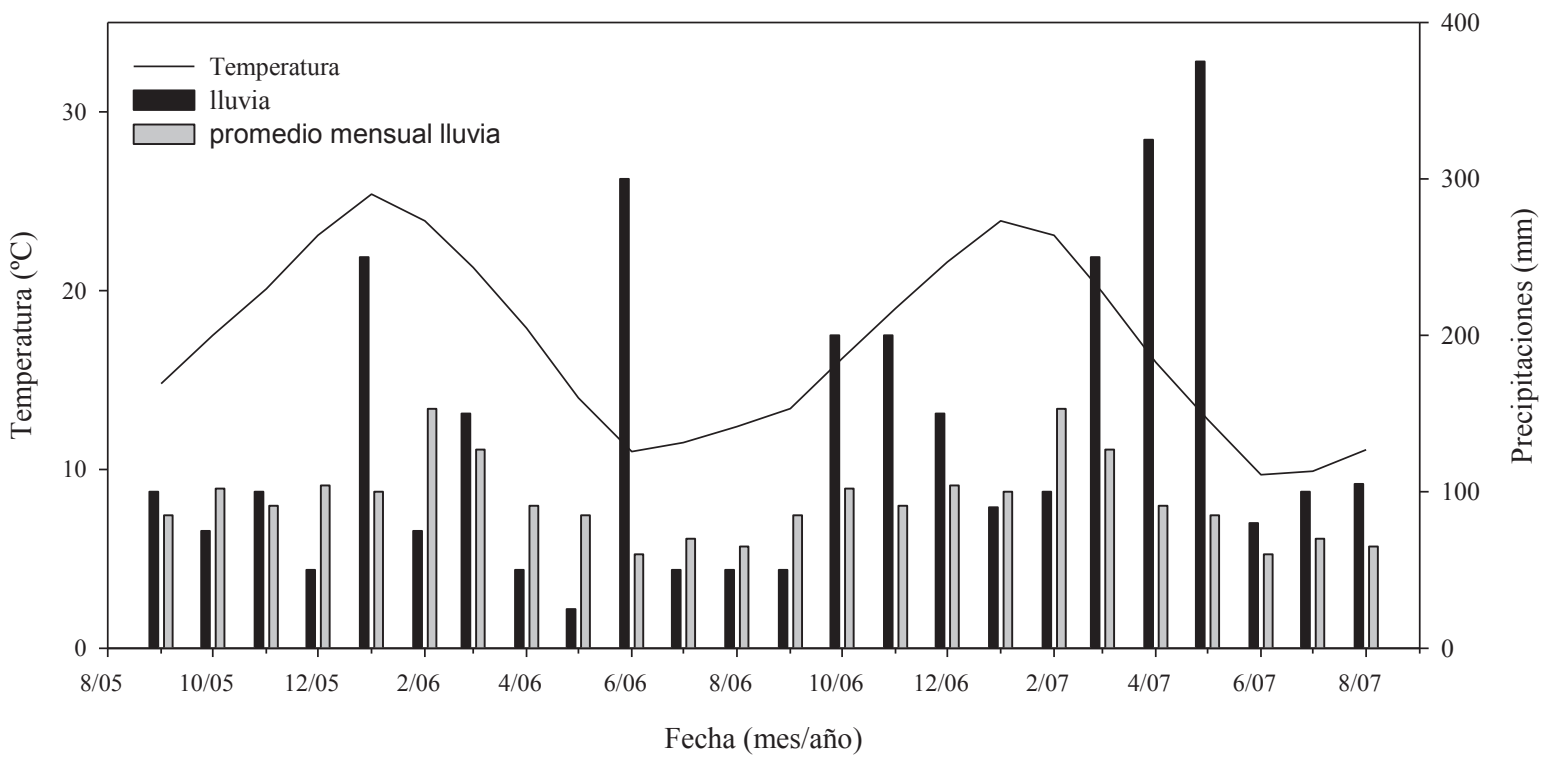

Figura 1. Evolución de las precipitaciones y temperaturas medias mensuales para el período de descomposición de restos de cosecha. Como referencia se incluye el promedio histórico de precipitaciones.

Precipitation and temperature evolution (monthly means) during the residues decomposition period. Reference to the historical average rainfall is included. 
Análisis químicos. Se realizó la molienda de los diferentes componentes de cosecha a tamaños de partículas menores a $0,5 \mathrm{~mm}$. Se determinó el contenido de fósforo, calcio, magnesio y potasio de las muestras de cada fracción, previa mineralización por vía seca, mediante calcinación de $1 \mathrm{~g}$ (ramas, hojas y corteza) o $2 \mathrm{~g}$ (madera) de muestra durante 5 horas a $550{ }^{\circ} \mathrm{C}$, y posterior disolución de las cenizas con $5 \mathrm{~mL}$ de $\mathrm{HCl}$ al $20 \%$. En el extracto se determinó el fósforo por colorimetría, calcio y magnesio por espectrofotometría de absorción atómica, y el potasio por espectrofotometría de emisión. La determinación del contenido de nitrógeno fue realizada mediante destilación Kjeldhal, previa mineralización de la muestra por vía húmeda $\left(\mathrm{H}_{2} \mathrm{SO}_{4}\right.$ y mezcla de $\mathrm{CuSO}_{4} / \mathrm{K}_{2} \mathrm{SO}_{4}$ ) a $370{ }^{\circ} \mathrm{C}$ durante 60 minutos.

El contenido de carbono total y soluble de las muestras de planta se analizó mediante oxidación con $\mathrm{K}_{2} \mathrm{Cr}_{2} \mathrm{O}_{7}$ en presencia de $\mathrm{H}_{2} \mathrm{SO}_{4}$ a $150{ }^{\circ} \mathrm{C}$ y posterior determinación colorimétrica. La determinación de lignina se realizó mediante hidrólisis ácida. Se realizó el análisis de polifenoles, mediante la extracción con $\mathrm{H}_{2} \mathrm{O}$ a $100{ }^{\circ} \mathrm{C}$ por una hora (Corbeels et al. 2003), y la determinación por colorimetría usando el reactivo Folin-Ciocalteu.

Índices de eficiencia de uso de nutrientes y modelo de descomposición de restos. Se calcularon tres índices de eficiencia de uso de nutrientes en la producción de biomasa: -Eficiencia de uso en producción total de biomasa $\left(\mathrm{EUN}_{1}\right)$ = kilogramo materia seca de biomasa total aérea producida por kilogramo de nutriente total absorbido (total de nutrientes en la biomasa aérea total).
-Eficiencia de uso en producción total de trozas $\left(\mathrm{EUN}_{2}\right)=$ kilogramo materia seca de biomasa total de trozas comerciales por kilogramo de nutriente total absorbido.

-Coeficiente de utilización biológica (CUB) = kilogramo de materia seca de madera producida (trozas comerciales) por kilogramo de nutrientes presentes en trozas.

Se calculó la tasa de descomposición de los diferentes residuos de cosecha a partir de la biomasa remanente luego de determinado tiempo usando un modelo de disminución exponencial (ecuación 1) del tipo:

$$
\mathrm{P} / \mathrm{P}_{\text {inic }}=\mathrm{e}^{-k t} \text {, siendo } \mathrm{P} / \mathrm{P}_{\text {inic }}
$$

Donde: $\mathrm{P} / \mathrm{P}_{\text {inic }}=$ fracción de material remanente al tiempo $t$ medido en años, y $k=$ constante de descomposición. A partir de este modelo se calculó la vida media en años de las diferentes fracciones.

El software usado fue CurveExpert Basic, de uso libre en la Web.

\section{RESULTADOS}

Extracción de nutrientes. El total de biomasa producida por el bosque fue de 137,5 $\mathrm{Mg} \mathrm{ha}^{-1}$, de los cuales el 77,5\% correspondieron a las trozas comerciales exportadas (cuadro 2), mientras que el restante $22,5 \%$ lo constituyen los residuos de la cosecha dejados en el sitio (corteza, ramas $\mathrm{y}$ hojas).

Cuadro 2. Producción de biomasa aérea, proporción de cada componente de cosecha, concentración de nutrientes de los componentes y nutrientes totales extraídos por componente de una plantación de Eucalyptus globulus de 10 años de edad (promedio de 24 árboles). Los valores entre paréntesis indican el desvío estándar.

Aerial biomass production, proportion of each harvest component, nutrient concentration of the components, and total nutrient extraction by component of a 10 year-old Eucalyptus globulus plantation (mean of 24 trees). Standard deviation values are indicated between parentheses.

\begin{tabular}{|c|c|c|c|c|c|c|c|c|c|c|c|c|}
\hline \multirow{2}{*}{$\begin{array}{l}\text { Componentes } \\
\text { de cosecha }\end{array}$} & \multirow{2}{*}{$\begin{array}{l}\text { Biomasa } \\
\mathrm{Mg} \mathrm{ha}^{-1}\end{array}$} & \multirow{2}{*}{$\begin{array}{c}\text { Biomasa } \\
\%\end{array}$} & \multirow{2}{*}{\multicolumn{5}{|c|}{$\begin{array}{l}N \\
\\
-\end{array}$}} & $\mathrm{N}$ & $\mathrm{P}$ & K & $\mathrm{Ca}$ & $\mathrm{Mg}$ \\
\hline & & & & & & & & \multicolumn{5}{|c|}{ - } \\
\hline Trozas & $\begin{array}{l}106,7 \\
(27,7)\end{array}$ & 77,5 & $\begin{array}{c}0,4 \\
(0,1)\end{array}$ & $\begin{array}{c}0,05 \\
(0,01)\end{array}$ & $\begin{array}{c}0,4 \\
(0,1)\end{array}$ & $\begin{array}{c}1,3 \\
(0,2)\end{array}$ & $\begin{array}{c}0,20 \\
(0,04)\end{array}$ & 43,8 & 5,4 & 34,6 & 138,3 & 25,8 \\
\hline Hojas & $\begin{array}{r}2,3 \\
(1,0)\end{array}$ & 1,7 & $\begin{array}{l}13,5 \\
(0,8)\end{array}$ & $\begin{array}{c}0,70 \\
(0,14)\end{array}$ & $\begin{array}{c}4,5 \\
(0,8)\end{array}$ & $\begin{array}{l}13,0 \\
(1,8)\end{array}$ & $\begin{array}{c}1,01 \\
(0,12)\end{array}$ & 31,7 & 1,8 & 10,3 & 29,6 & 2,3 \\
\hline Ramas finas & $\begin{array}{r}1,3 \\
(0,6)\end{array}$ & 0,9 & $\begin{array}{c}6,7 \\
(0,9)\end{array}$ & $\begin{array}{c}0,07 \\
(0,01)\end{array}$ & $\begin{array}{c}4,6 \\
(0,9)\end{array}$ & $\begin{array}{l}16,4 \\
(2,3)\end{array}$ & $\begin{array}{c}0,91 \\
(0,14)\end{array}$ & 8,8 & 0,1 & 6,3 & 21,0 & 1,1 \\
\hline Ramas medias & $\begin{array}{r}1,5 \\
(0,6)\end{array}$ & 1,1 & $\begin{array}{c}4,2 \\
(0,4)\end{array}$ & $\begin{array}{c}0,19 \\
(0,03)\end{array}$ & $\begin{array}{c}4,5 \\
(0,8)\end{array}$ & $\begin{array}{c}9,9 \\
(1,4)\end{array}$ & $\begin{array}{l}1,71 \\
(0,42)\end{array}$ & 6,3 & 0,3 & 6,9 & 14,6 & 2,5 \\
\hline Ramas gruesas & $\begin{array}{l}10,0 \\
(4,4)\end{array}$ & 7,3 & $\begin{array}{c}1,2 \\
(0,2)\end{array}$ & $\begin{array}{c}0,05 \\
(0,02)\end{array}$ & $\begin{array}{c}3,5 \\
(0,8)\end{array}$ & $\begin{array}{c}7,9 \\
(1,5)\end{array}$ & $\begin{array}{c}0,90 \\
(0,15)\end{array}$ & 11,1 & 0,5 & 34,6 & 78,3 & 8,9 \\
\hline Corteza & $\begin{array}{l}15,8 \\
(4,7)\end{array}$ & 11,5 & $\begin{array}{c}2,3 \\
(0,2)\end{array}$ & $\begin{array}{c}0,25 \\
(0,05)\end{array}$ & $\begin{array}{c}5,5 \\
(1,4)\end{array}$ & $\begin{array}{l}30,0 \\
(2,4)\end{array}$ & $\begin{array}{c}2,40 \\
(0,53)\end{array}$ & 36,3 & 4,0 & 87,1 & 479,6 & 37,9 \\
\hline Total cosecha & 137,5 & 100,0 & & & & & & 138,0 & 12,1 & 179,8 & 761,4 & 78,5 \\
\hline
\end{tabular}


La concentración de elementos nutritivos varió según el componente del árbol, siendo las trozas quienes presentaron las menores concentraciones para todos los elementos (cuadro 2). Las hojas mostraron las mayores concentraciones de nitrógeno y fósforo, mientras que la corteza presentó los mayores valores de magnesio y particularmente calcio. Por último, el potasio presentó valores similares entre los distintos componentes de los residuos, siendo igualmente la corteza quien tuvo la mayor concentración. El calcio fue el nutriente que presentó mayor variabilidad dentro del árbol, con concentraciones que van desde $30,0 \mathrm{~g}$ $\mathrm{kg}^{-1}$ en la corteza hasta valores 24 veces inferiores en trozas $\left(1,3 \mathrm{~g} \mathrm{~kg}^{-1}\right)$. Por su parte, el fósforo presentó valores similares para los distintos componentes, con excepción de hojas en donde los contenidos fueron superiores.

Tomando en cuenta las cantidades totales de elementos absorbidas por los árboles se observaron variaciones importantes. El calcio presentó la extracción absoluta mayor, aunque solo un $18 \%$ de esta cantidad fue exportada por las trozas, permaneciendo el remanente sobre todo en la corteza (63\%) (cuadro 2). El fósforo presentó las menores cantidades extraídas, aunque al mismo tiempo fue el nutriente con mayor porcentaje de exportación en las trozas, seguido en orden creciente por magnesio, nitrógeno, potasio y calcio.

Para los elementos nutritivos considerados, y en esta especie de Eucalyptus, en los 10 años del turno se exportaron por las trozas menos del $45 \%$ del total de cada nutriente extraído.

En los residuos, la mayor proporción de los elementos nutritivos se encontraron en corteza y hojas. En la corteza se encontraron sobre todo los mayores porcentajes de cationes. Las ramas presentaron contenidos bajos de potasio, excepto las ramas gruesas con un $19 \%$ del total extraído. Debe de tenerse en cuenta que en las ramas se incluía la corteza correspondiente, por lo que su contenido en nutrientes es relativamente importante comparado con los materiales leñosos como trozas.

Eficiencia de uso de los nutrientes. Los índices de eficiencia correspondientes a los 24 árboles evaluados variaron según el nutriente en consideración, aunque esto se relaciona inversamente con las concentraciones de los mismos en el suelo (cuadro 3).

Descomposición de restos de cosecha. Se observó una disminución en la biomasa de los distintos componentes de cosecha de E. globulus durante los 24 meses posteriores a la misma (figura 3). Si bien se observa una tendencia a la disminución de la biomasa en todos los restos, existen diferencias en las tasas de descomposición de cada uno de ellos, siendo las hojas quienes mostraron la mayor pérdida. La descomposición acumulada de las diferentes fracciones a los 24 meses post-cosecha indica que fue degradado el $68 \%$ de las hojas, el $31 \%$ de las ramas finas, el $28 \%$ de las ramas medias y el $19 \%$ de la corteza. El $30 \%$ del total de los residuos (promedio ponderado según pesos totales de cada fracción al momento de la cosecha) se degradó en los dos años posteriores a la cosecha.

Se observaron distintos contenidos iniciales de carbono total en los diferentes componentes (cuadro 4), donde las hojas mostraron las más altas concentraciones, mientras que la corteza presentó los contenidos más bajos. Los

Cuadro 3. Índices de eficiencia de uso de elementos nutritivos para Eucalyptus globulus (promedio de 24 árboles).

Nutritious elements utilization efficiency indexes for Eucalyptus globulus (average of 24 trees).

\begin{tabular}{crrrrr}
\hline \multirow{2}{*}{ Índice $^{\dagger}$} & \multicolumn{5}{c}{ Eficiencia de uso de los elementos nutritivos } \\
& \multicolumn{1}{c}{$\mathrm{N}$} & \multicolumn{1}{c}{$\mathrm{P}$} & \multicolumn{1}{c}{$\mathrm{K}$} & $\mathrm{Ca}$ & \multicolumn{1}{c}{$\mathrm{Mg}$} \\
\hline & 572 & 6.567 & 439 & 104 & 1.003 \\
$\mathrm{EUN}_{1}$ & 420 & 4.819 & 322 & 76 & 736 \\
$\mathrm{EUN}_{2}$ & 1.322 & 10.670 & 1.672 & 418 & 2.239 \\
$\mathrm{CUB}$ & &
\end{tabular}

†UUN1: eficiencia de uso en producción total de biomasa. $\mathrm{EUN}_{2}=$ eficiencia de uso en producción total de trozas. CUB: coeficiente de utilización biológica.

Cuadro 4. Contenido de carbono total, carbono soluble, relación carbono: nitrógeno, polifenoles solubles y lignina de restos de Eucalyptus globulus inmediatamente después de la cosecha y luego de 12 meses.

Total carbon, soluble carbon, carbon: nitrogen ratio, soluble polyphenols, and lignin content of Eucalyptus globulus residues immediately after harvest and 12 months later.

\begin{tabular}{|c|c|c|c|c|c|c|c|c|}
\hline \multirow{3}{*}{ Componente } & C total & C soluble & $\mathrm{C}: \mathrm{N}$ & $\begin{array}{c}\text { Polifenoles } \\
\text { solubles }\end{array}$ & Lignina & C soluble & $\begin{array}{c}\text { Polifenoles } \\
\text { solubles }\end{array}$ & Lignina \\
\hline & \multicolumn{5}{|c|}{------------------------ Mes 0 -------------------------- } & \multicolumn{3}{|c|}{------------ Mes 12 ------------ } \\
\hline & $\mathrm{g} \mathrm{kg}^{-1}$ & $\mathrm{~g} \mathrm{~kg}^{-1}$ & & $\mathrm{~g} \mathrm{~kg}^{-1}$ & $\%$ & $\mathrm{~g} \mathrm{~kg}^{-1}$ & $\mathrm{~g} \mathrm{~kg}^{-1}$ & $\%$ \\
\hline Corteza & 462 & 84 & 243 & 41,4 & 36,2 & 28 & 29,3 & 42,8 \\
\hline Hoja & 569 & 143 & 42 & 91,4 & 30,6 & 47 & 17,3 & 39,5 \\
\hline Rama fina & 517 & 66 & 144 & 67,2 & 28,5 & 34 & 16,1 & 31,1 \\
\hline Rama media & 493 & 28 & 411 & 25,8 & 24,4 & 17 & 6,6 & 25,9 \\
\hline
\end{tabular}




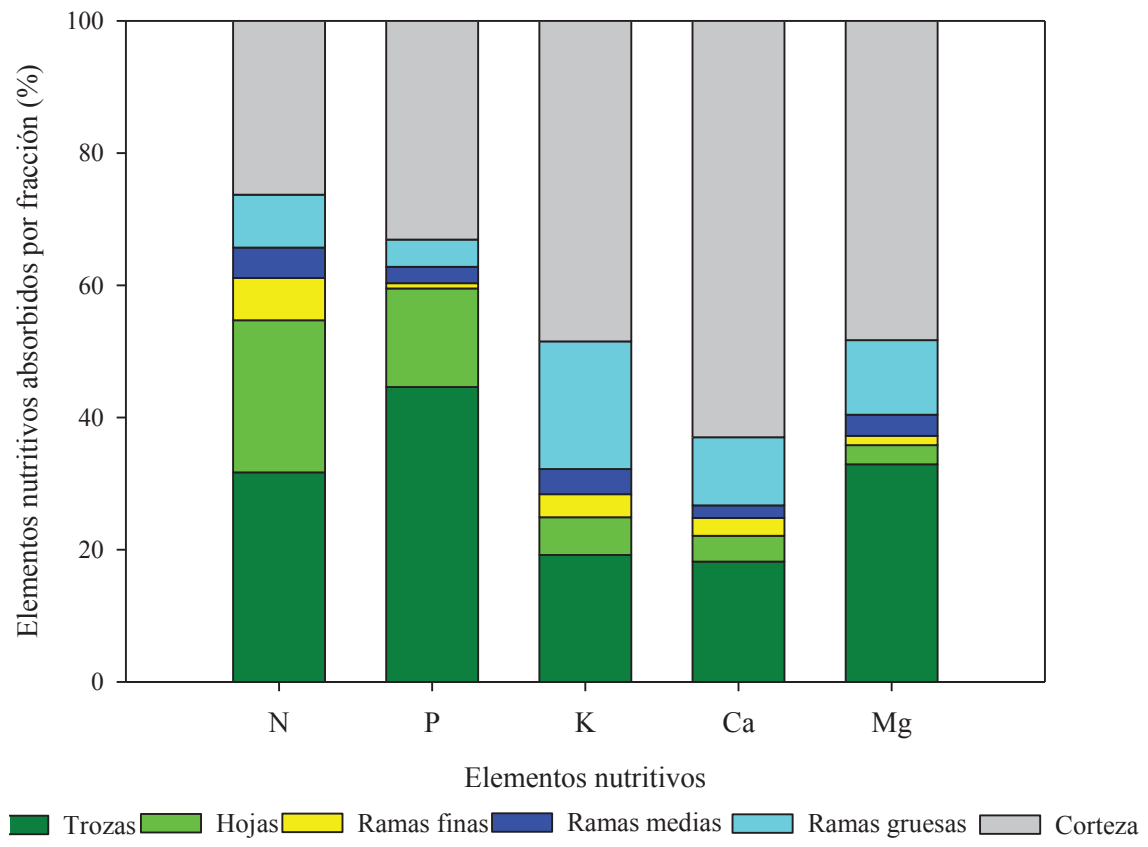

Figura 2. Porcentaje de los elementos nutritivos totales absorbidos en los diferentes componentes de la cosecha de una plantación de Eucalyptus globulus de 10 años. Valores promedios de 24 árboles.

Percentage of the total absorbed nutritious elements in the different harvest components of a 10 year-old Eucalyptus globulus plantation. Mean values of 24 trees.

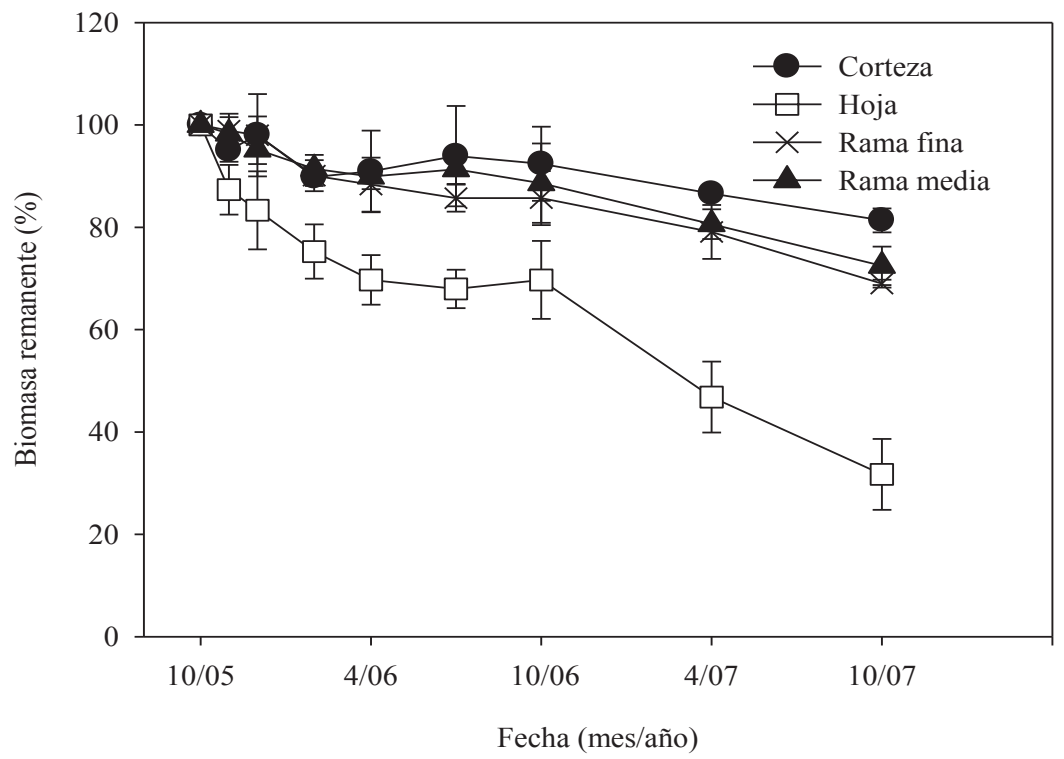

Figura 3. Evolución de la biomasa remanente de las distintas fracciones de cosecha de Eucalyptus globulus, expresada como porcentaje de la biomasa inicial. Las barras verticales indican desvío estándar.

Time course of the remaining biomass of the different fractions after the Eucalyptus globulus harvest, expressed as a percentage of the initial biomass. Vertical bars indicate standard deviation.

contenidos de carbono soluble presentaron los valores mayores en hojas, y los menores en ramas gruesas. Otro parámetro relacionado con la velocidad de descomposición, la relación carbono: nitrógeno de los restos, presentó valores extremadamente altos para ramas y corteza.
El modelo de decrecimiento exponencial de biomasa para cada componente de cosecha, muestra constantes de descomposición diferente para cada resto (cuadro 5), siendo más elevada para hojas. En cuanto a la vida media calculada, la corteza es el componente que mostró mayores 
Cuadro 5. Pérdida porcentual de biomasa en el período de dos años, constante de descomposición (k), $r^{2}$ del modelo y vida media de los restos de cosecha de Eucalyptus globulus.

Biomass lost in percentage over the two year-period, decomposition constant $(\mathrm{k}), r^{2}$ of the model, and mean life of the Eucalyptus globulus harvest residues.

\begin{tabular}{lcccc}
\hline Componente & $\%$ pérdida & $\mathrm{k}$ & $\mathrm{r}^{2}$ & $\mathrm{t}_{1 / 2}$ \\
\hline & & $\left(\right.$ año $\left.{ }^{-1}\right)$ & & (años) \\
\hline Corteza & 18,6 & 0,08 & 0,90 & 8,49 \\
Hoja & 68,3 & 0,50 & 0,94 & 1,40 \\
Rama fina & 31,0 & 0,17 & 0,96 & 4,07 \\
Rama media & 27,5 & 0,14 & 0,95 & 4,81 \\
\hline
\end{tabular}

valores. Esto se explica por el mayor tamaño de esta fracción, así como por la mayor relación carbono: nitrógeno respecto a las restantes fracciones.

Los valores de descomposición observados se refieren a restos en contacto con el suelo, siendo complejo estimar qué proporción de ellos quedará directamente sobre el suelo y qué proporción lo hará separada de este por otra capa de restos, lo que retrasaría la descomposición.

La evolución del contenido de elementos nutritivos en los distintos componentes de cosecha mostró diferencias de comportamiento entre los nutrientes en los restos en el primer año (figuras 4 y 5). En el segundo año aumentó la descomposición de los restos, y, por tanto, también ocurrió una disminución en las cantidades totales de todos los nutrientes.
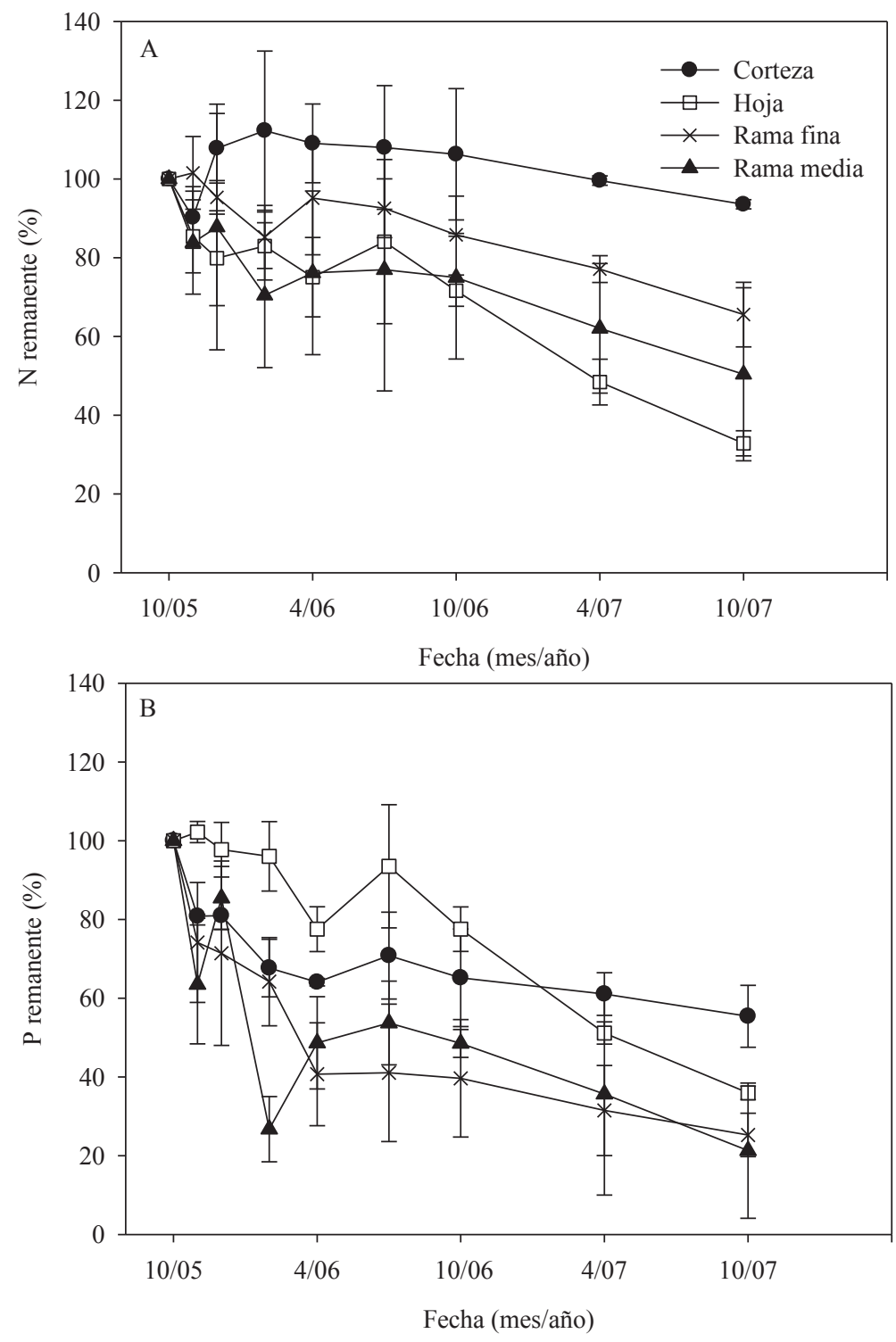

Figura 4. Evolución de los contenidos remanentes de (A) nitrógeno y (B) fósforo en restos de cosecha de Eucalyptus globulus durante el proceso de descomposición. Las barras verticales indican desvío estándar.

Time courses of the remains contents of (A) nitrogen and (B) phosphorus in harvest residues of Eucalyptus globulus during the decomposition process. Vertical bars indicate standard deviation. 

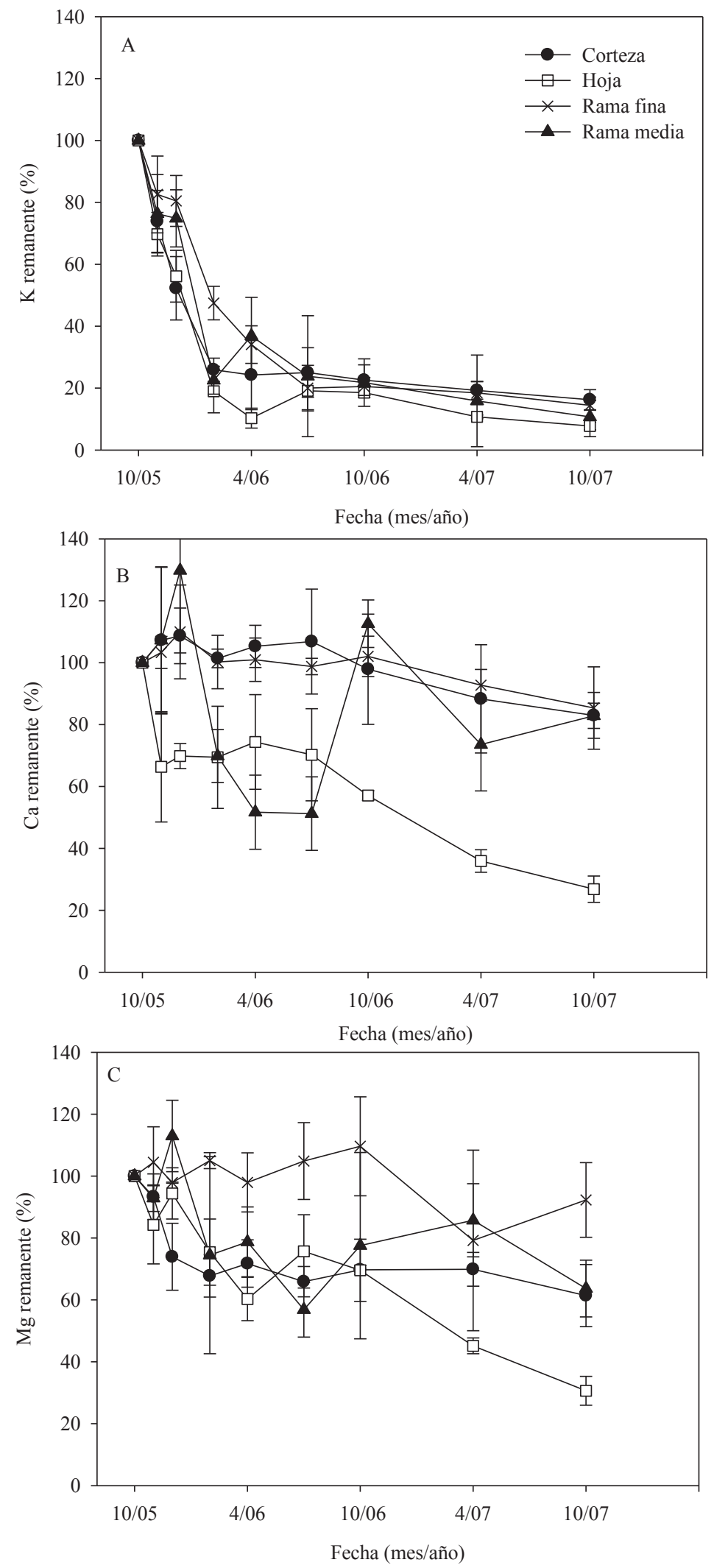

Figura 5. Evolución de los contenidos remanentes de (A) potasio, (B) calcio y (C) magnesio en restos de cosecha de Eucalyptus globulus durante el proceso de descomposición. Las barras verticales indican desvío estándar.

Time courses of the remains contents of (A) potassium, (B) calcium and (C) magnesium in harvest residues of Eucalyptus globulus during the decomposition process. Vertical bars indicate standard deviation. 
Con respecto al nitrógeno, luego de una disminución en el primer mes, en la corteza se incrementó la cantidad total, lo que indicaría inmovilización neta de nitrógeno del suelo por parte de los microorganismos, debido a la pérdida proporcionalmente mayor de carbono y otros nutrientes (figura 4A). Luego del primer año, la descomposición de estos restos fue mayor, lo que llevó a una disminución del contenido total de este nutriente. En hojas, ramas finas y medias, las concentraciones de nitrógeno se mantuvieron en los restos más o menos constantes a lo largo de los dos años, siendo la degradación de la biomasa en cada caso quien determinó la disminución en las cantidades totales de este nutriente.

El fósforo presentó una lenta disminución en las cantidades totales en el primer año en casi todas las fracciones. En las hojas se observó una pérdida de fósforo, a pesar de que su concentración sufrió un aumento en los primeros muestreos (datos no presentados). Para ramas finas y medias, las concentraciones se mantuvieron constantes, con lo cual las pérdidas del nutriente se explican por la propia degradación de la fracción (figura 4B). La corteza presentó una disminución en las cantidades en los primeros seis meses, explicado por una ligera disminución en la concentración en sus restos $\left(0,05 \mathrm{~g} \mathrm{~kg}^{-1}\right.$ en el inicio del experimento a $0,04 \mathrm{~g} \mathrm{~kg}^{-1}$ en el resto de las evaluaciones, datos no presentados). En el segundo año, la disminución del nutriente acompañó la degradación de los restos en todos los casos. Es importante destacar que todas las fracciones presentaron muy pequeñas concentraciones de este nutriente (entre 0,9 y $0,04 \mathrm{~g} \mathrm{~kg}^{-1}$, según la fracción y muestreo).

La pérdida de potasio de todos los componentes de cosecha fue muy rápida (alrededor del $80 \%$ del potasio contenido en hoja y corteza en los cuatro primeros meses del período estudiado) (figura 5A).

Con respecto a calcio, todas las fracciones mantuvieron o aumentaron ligeramente sus concentraciones con el tiempo, indicando esto que la pérdida de este nutriente fue menor respecto a la de biomasa. En la corteza, las concentraciones superaron los $30 \mathrm{~g} \mathrm{~kg}^{-1}$ en todos los muestreos, lo que se vio reflejado en la alta proporción del calcio inicialmente presente observada al final del período de descomposición (figura 5B).

Finalmente, la pérdida de magnesio ocurrió en forma lenta a partir de la cosecha para todos los residuos (figura 5C). Las concentraciones de las distintas fracciones permanecieron más o menos constantes, por lo que la evolución de la cantidad de magnesio de los restos se relacionó estrechamente con la pérdida de biomasa. Solamente en las hojas se produjeron pérdidas mayores de magnesio que pasaron probablemente a formas minerales solubles.

\section{DISCUSIÓN}

El $77,5 \%$ de la biomasa total producida corresponde a la madera comercial, mientras que el restante $22,5 \%$ lo constituyen los residuos de cosecha, siendo la corteza el
$11,5 \%$ de la biomasa total. Este componente de restos de cosecha presenta altas concentraciones de elementos nutritivos, sobre todo cationes $(49,63$ y $48 \%$ del potasio, calcio y magnesio total extraído, respectivamente). En esa línea, Santana et al. (2000) en Brasil, indicaron que aunque la corteza de distintas especies de Eucalyptus representó solo entre el 10 y $18 \%$ del total de la biomasa aérea cosechada, presentó un alto contenido de elementos nutritivos $(24,46,41,73$ y $65 \%$ del nitrógeno, fósforo, potasio, calcio y magnesio total extraído, respectivamente). Estos autores concluyen que el descortezado en el campo evita la exportación del 14, 27, 22, 58 y $47 \%$ del nitrógeno, fósforo, potasio, calcio y magnesio total extraído, respectivamente, para E. grandis y E. saligna. En dicho estudio el efecto del sitio fue más importante que la diferencia en material genético. Por su parte, Laclau et al. (2000), evaluando clones de Eucalyptus spp. en el Congo, destacan también la importancia de dejar la corteza en el sitio, lo cual reduce la exportación de calcio y magnesio en más del $50 \%$. Hernández et al. (2009), en plantaciones de Uruguay, encontraron también que la corteza contenía una alta proporción de los nutrientes extraídos, concluyendo sobre la importancia del descortezado en el sitio de cosecha.

Los contenidos de elementos nutritivos y su distribución entre los distintos componentes del árbol varían para las distintas especies de Eucalyptus (Grove et al. 1996). Wise y Pitman (1981) encontraron que para seis especies de Eucalyptus en Australia el porcentaje de dichos elementos presentes en los troncos de plantaciones de 10 años fue diferente según el nutriente, donde el menos variable fue el nitrógeno (43 a $57 \%$ ), y el más variable calcio (de 9 a $61 \%)$. Los autores destacan también los elevados contenidos de calcio en las especies de corteza lisa, donde llega a constituir entre el 42 y $47 \%$ del total de los nutrientes de la planta.

En el experimento, todos los elementos nutritivos presentan un porcentaje potencial de retorno a través de los restos de cosecha mayor al $55 \%$, siendo potasio y calcio los de mayor retorno, este último explicado por su alta proporción en la corteza. Los porcentajes de reciclaje potencial se encuentran, por lo general, dentro de los rangos mencionados por Vaz de Arruda Silveira y Higashi (2002) para E. urograndis y E. grandis en Brasil, a excepción del potasio que presenta un valor algo superior $(8 \%)$ y el magnesio que presenta un valor $20 \%$ menor respecto a lo mencionado por estos autores.

Respecto a las concentraciones de elementos nutritivos en las diferentes partes de la planta, existe concordancia entre este estudio y los rangos presentados en el trabajo de Judd et al. (1996), excepto, para calcio donde las concentraciones encontradas en este estudio en corteza y hoja, y en cierta medida en la madera, superan los rangos citados por estos autores. Respecto a magnesio fueron encontradas concentraciones en la madera algo superiores al valor máximo de los rangos citados por este autor. Esto puede explicarse por los altos contenidos en estos elemen- 
tos que presentaba el suelo donde se ubicó el experimento (cuadro 1).

En referencia a la eficiencia de uso de los elementos nutritivos para E. globulus, se encuentran valores de $\mathrm{EUN}_{1}$ muy altos para fósforo $(6.567 \mathrm{~kg}$ de biomasa aérea total por kilogramo de nutriente absorbido), alto para magnesio (1.003), medios para nitrógeno y potasio (572 y 439, respectivamente) y bajos para el calcio (104). Estas eficiencias están relacionadas con las concentraciones de estos elementos en el suelo, que para el experimento fueron altas para calcio y demás bases, y muy bajas para el fósforo (cuadro 1). Santana et al. (2000), en estudios realizados para diferentes especies de Eucalyptus en varios estados de Brasil, con distintas condiciones climáticas y edáficas (generalmente suelos de baja fertilidad), obtuvieron valores promedio de índices de eficiencia de uso muy elevados para fósforo y magnesio $(5.319$ y $3.095 \mathrm{~kg}$ de biomasa aérea total por kilogramo de nutriente absorbido, respectivamente) y menores para los nutrientes potasio, nitrógeno y calcio $(822,589$ y $513 \mathrm{~kg}$ de biomasa aérea total por kilogramo de nutriente absorbido, respectivamente). Valores del mismo orden a los anteriores fueron encontrados por Morais et al. (1990) y Herbert (1996), para diferentes especies de Eucalyptus de 8 años de edad en Brasil, y de siete años de edad en Sudáfrica, respectivamente. Por el contrario, Laclau et al. (1999), en plantaciones híbridas de E. urograndis en el Congo, encontraron eficiencias de uso menores para el fósforo y más altas para los nutrientes potasio, calcio y magnesio, mientras que para nitrógeno obtuvieron resultados similares a los de nuestro trabajo.

En cuanto a las medidas de eficiencia a través del coeficiente de utilización biológica, Freitas Melo et al. (1995) obtuvieron para E. saligna de siete años en distintos sitios de Río Grande del Sur (Brasil), índices de coeficiente de utilización biológica promedios muy altos para los nutrientes fósforo y magnesio (7.490 y $4.126 \mathrm{~kg}$ de materia seca de madera producida por kilogramo de nutrientes presentes en trozas, respectivamente), y menores para los nutrientes potasio y calcio $(933$ y $931 \mathrm{~kg}$ de materia seca de madera producida por kilogramo de nutrientes en trozas, respectivamente), lo que está en relación inversa con la concentración de dichos nutrientes en la madera. En nuestro estudio los valores de coeficiente de utilización biológica encontrados fueron superiores respecto al trabajo antes mencionado para fósforo y potasio, e inferiores para calcio y magnesio (cuadro 3). Estas diferencias pueden explicarse por los bajos contenidos en fósforo y altos para cationes, particularmente calcio y magnesio, que presentan los suelos de Uruguay en general, y el del experimento en particular.

La degradación de los restos es variable según la fracción, lo que se explica por el diferente tamaño de los componentes, puesto que aquellos con mayor tamaño (corteza, ramas medias, ramas finas) son los de más lenta descomposición. Además, la mayor pérdida de peso que sufrieron las hojas en los seis primeros meses podría relacionarse con la degradación de sus componentes más inestables, como compuestos orgánicos solubles o de estructura química más lábil para la degradación microbiana, quedando como remanentes los constituyentes más resistentes a la descomposición. Una estimación de estas sustancias más lábiles es el contenido de carbono orgánico soluble, el cual es claramente mayor en hojas en comparación al del resto de los componentes (cuadro 4). Resultados de experimentos de incubación en condiciones controladas para E. grandis en Brasil indicaron también mayores contenidos de carbono orgánico soluble en hojas respecto a otros residuos, lo que estuvo asociado a mayores tasas anuales de descomposición (0,59 años) para las hojas (Rezende et al. 2001). Hernández et al. (2009), estudiando la descomposición de residuos de cosecha de $E$. dunnii de 9 años de edad, encontraron mayores contenidos de carbono soluble en hojas respecto a las otras componentes de los residuos (ramas, despuntes y corteza), con una tasa de descomposición anual muy superior $(0,92$ años) comparada con las otras fracciones (3,51 años en ramas, 3,66 años en despuntes y 5,27 años en corteza). El presente estudio determina tasas de descomposición menores respecto al trabajo de Hernández et al. (2009) (cuadro 5). Estos resultados se alinean con diversos estudios que determinan mayor perdurabilidad de los restos de E. globulus en relación a otras especies como E. dunnii (Sánchez 2011).

También es muy importante considerar que en la primera etapa la degradación microbiana tiene un peso menor respecto a organismos de la macro y meso fauna que son los que mastican y digieren los componentes más palatables y de menor cantidad de inhibidores.

El contenido de nitrógeno y la relación carbono: nitrógeno se han considerado muy importantes en la velocidad de descomposición de los materiales vegetales (Burgess et al. 2002), dado que los microorganismos, si bien basan su actividad en la disponibilidad de carbono, necesitan cierta cantidad de los demás nutrientes, y ante la escasez de los mismos se enlentece la descomposición (Mary et al. 1996). Esto pasa a ser muy importante para restos de gran volumen y poco contacto con el suelo, ya que cuando los restos son incorporados, el nitrógeno mineral del suelo es inmovilizado por la biomasa microbiana, y de este modo el suelo provee el nitrógeno necesario para el crecimiento de la misma (Ambus y Jenssen 2001). Los resultados de nuestro trabajo concuerdan con lo expresado, ya que las hojas -que fue la fracción que más se degradó durante el estudio- es la que presentó menores relaciones carbono: nitrógeno, siendo entonces probablemente las proveedoras de parte del nitrógeno necesario a los microorganismos para su descomposición.

Diversos estudios (Lovett et al. 2004, Verkaik et al. 2006) determinaron también que los polifenoles reducen la actividad microbiana, además de producir cambios en la comunidad de los microorganismos, ya sea por toxicidad o por inhibición, lo que traería como consecuencia una disminución en la degradación de los restos. Duran- 
te el primer año del estudio de degradación post-cosecha se perdieron gran parte de los polifenoles, lo que podría explicar en parte el importante aumento en la descomposición que tuvieron todos los restos en el segundo año de la investigación. Por otra parte, el cuadro 4 muestra el incremento porcentual de lignina en el primer año posterior a la cosecha para todos los restos, lo cual se explica por la mayor degradación de los componentes más inestables, como compuestos orgánicos solubles quedando como remanentes los materiales más resistentes a la descomposición, como la lignina.

Los aportes de elementos nutritivos por parte de los residuos forestales son variables tanto en cantidad como en la eficiencia de uso posterior por el bosque. Para el nitrógeno pueden producirse pérdidas durante la descomposición de los residuos, así como también de formas minerales resultantes de los procesos de mineralización. El fósforo podrá estar disponible dependiendo de procesos biológicos y químicos complejos. Los cationes (potasio, calcio y magnesio) vuelven al suelo, pudiendo sobre todo el potasio ser reutilizado en forma casi inmediata en la medida que su liberación a partir de los restos se da en los primeros meses. La lenta descomposición de algunos restos puede ser muy buena considerando que así podrá el bosque futuro absorberlos en el mediano y largo plazo, a medida que las plantas vayan creciendo. Además de mantener una reserva de elementos nutritivos en el sitio, los restos forestales aportan otros efectos, como ser, la reducción de pérdidas de agua por evaporación, contribuir en la mitigación de las pérdidas de suelo por erosión, ayudar al mantenimiento de la actividad biológica del suelo, como también limitar el desarrollo de malezas del suelo.

\section{CONCLUSIONES}

Si bien la exportación de biomasa de la especie bajo estudio (Eucalyptus globulus) resulta ser del orden del $78 \%$, los elementos nutritivos exportados con ella fuera del sitio significan un porcentaje relativamente bajo del total absorbido por la plantación (menos del $45 \%$ ), lo cual asegura que la mayor parte de los elementos absorbidos por las plantas permanecen en el sitio. Es importante destacar además que la práctica del descortezado en el campo previene de una elevada exportación de dichos elementos, dadas las altas concentraciones de calcio, magnesio y potasio en la corteza de esta especie.

El retorno de los elementos nutritivos al suelo y sus posibilidades de ser utilizados por una replantación del monte es dependiente de las tasas de descomposición de los restos de cosecha, las cuales son variables según el tamaño y composición de los restos. Si bien el promedio de pérdida de biomasa pos cosecha luego de dos años vía descomposición de restos es del $30 \%$, las menores tasas de descomposición son observadas en corteza y ramas, por tratarse de restos gruesos con altas relaciones carbono: nitrógeno, contenido de lignina o polifenoles.

\section{AGRADECIMIENTOS}

Los autores agradecen a CSIC (Universidad de la República Oriental del Uruguay) y empresas forestales (Montes del Plata, UPM, Weyerhaeuser, COFUSA y FYMNSA) la financiación de los estudios realizados.

\section{REFERENCIAS}

Ambus P, E Jenssen. 2001. Crop residue management strategies to reduce $\mathrm{N}$ losses - Interaction with crop N supply. Communications in Soil Science and Plant Analysis 32(7-8): 981-966.

Ankila J, L Hiremath, J Ewel, TG Cole. 2002. Nutrient use efficiency in three fast-growing tropical trees. Forest Science 48(4): 662-672.

Ankila J, L Hiremath, JJ Ewel. 2001. Ecosystem nutrient use efficiency, productivity, and nutrient accrual in model tropical communities. Ecosystems 4: 669-682.

Burgess MS, GR Mehuys, CA Madramootoo. 2002. Nitrogen dynamics of decomposing corn residue components under three tillage systems. Soil Science Society of America Journal 66(4): 1350-1358.

Corbeels M, AM O'Connell, TS Grove, DS Mendham, SJ Rance. 2003. Nitrogen release from eucalypt leaves and legume residues as influenced by their biochemical quality and degree of contact with soil. Plant and Soil 250(1): 15-28.

Freitas Melo V, R Ferreira de Novais, NF Félix de Barros, M Ferreira, L Da Costa. 1995. Balanço nutricional, eficiência de utilizaçao e avaliaçao da fertilidade do solo en P, K, Ca e $\mathrm{Mg}$ em plantios de Eucalipto no Río Grande do Sul. Revista IPEF 48/49: 8-16.

Gonçalves JLM, F Poggiani, JL Stape, MI Serrano, SL Mello, KC Mendez. 1997. Efeito de práticas de cultivo mínimo e intensivo do solo sobre a ciclagem de nutrientes, fertilidade do solo, configuraçao do sistema radicular e nutriçao mineral de povoamentos de Eucalyptus grandis. Piracicaba, Brasil. Relatorio final de Pesquisa, FAPESP, processo $\mathrm{N}^{\circ}$ 1994/4248-4.

Grove TS, BD Thomson, N Malajczuk. 1996. Nutritional physiology of Eucalyptus: uptake, distribution and utilization. In Attiwill PM, A Mark eds. Nutrition of Eucalypts. Collinwood, Australia. CSIRO Publishing. p. 77-108.

Hernández J, A Del Pino, L Salvo, G Arrarte. 2009. Nutrient export and harvest residue decomposition patterns of a $\mathrm{Eu}$ calyptus dunnii Maiden plantation in temperate climate of Uruguay. Forest Ecology and Management 258(2): 92-99.

Herbert MA. 1992. Nutrition of Eucalyptus in South Africa. Pietermaritzburg, South Africa. Bulletin Series 27/92. Institute for Comercial Forestry Research. 25 p.

Herbert MA. 1996. Fertilizers and eucalypt plantations in South Africa. In Attiwill PM, A Mark eds. Nutrition of Eucalypts. Collinwood, Australia. CSIRO Publishing. p. 303-325.

Judd TS, PM Attiwill, MA Adams. 1996. Nutrient concentrations in Eucalyptus: A synthesis in relation

to differences between taxa, sites and components. In Attiwill PM, A Mark eds. Nutrition of Eucalypts. Collinwood, Australia. CSIRO Publishing. p. 123-153.

Laclau JP, JP Bouillet, J Ranger. 2000. Dynamics of biomass and nutrient accumulation in a clonal plantation of Eucalyptus 
in Congo. Forest Ecology and Management 128(3):181196.

Lovett GM, KC Weathers, M Arthur, JC Schultz. 2004. Nitrogen cycling in a northern hardwood forest: Do species matter? Biogeochemistry 67(3):289-308.

Mary B, S Recous, D Darwis, D Robin. 1996. Interactions between decomposition of plant residues and nitrogen cycling in soil. Plant and Soil 181(1):71-82.

Morais EJ, NF Barros, RF Novais, R Brandi. 1990. Biomassa e eficiência nutricional de espécies de eucalipto em duas regiões bioclimáticas de Minas Gerais. Revista Brasileira de Ciência do Solo 14(3): 353-362.

Olson JS. 1963. Energy storage and the balance of producers and decomposers in ecological systems. Ecology 44: 322-331.

Palviainen M, L Finér, A Kurka, H Mannerkoski, S Piirainen. 2004. Release of potassium, calcium, iron and aluminum from Norway spruce, Scots pine and silver birch logging residues. Plant and Soil 259(1-2): 123-136.

Rezende JL, QS Garcia, MR Scotti. 2001. Laboratory decomposition of Dalbergia nigra All. ex Benth and Eucalyptus grandis W. Hill ex Maiden leaves in forest and eucalypt plantation soils. Acta Botánica Brasílica 15(3): 305-312.

Ribeiroa C, M Madeirab, MC Araujo. 2002. Decomposition and nutrient release from leaf litter of Eucalyptus globulus grown under different water and nutrient regimes. Forest Ecology and Management 171: 31-41.

Sánchez G. 2011. Descomposición de restos de cosecha de Eucalyptus sp. y Pinus taeda en condiciones controladas de humedad y temperatura. Tesis de Maestría. Montevideo. Uruguay. Facultad de Agronomía, Universidad de La República. $84 \mathrm{p}$.
Santana RC, NF Barros, NB Comerford. 2000. Above-ground biomass, nutrient content, and nutrient use efficiency of eucalypt plantations growing in different sites in Brazil. New Zealand Journal of Forestry Science 30(1-2): 225-236.

Shammas K, AM O'Connell, TS Grove, R McMurtrie, P Damon, SJ Rance. 2003. Contribution of decomposing harvest residues to nutrient cycling in a second rotation Eucalyptus globulus plantation in south-western Australia. Biology and Fertility of Soils 38(4): 228-235.

Smith CT, AT Lowe, PN Beets. 1994. Nutrient accumulation in second-rotation Pinus radiata after harvest residue management and fertilizer treatment of coastal sand dunes. New Zealand Journal of Forestry Science 24(2-3): 362-389.

Spangenberg A, U Grimm, JR Silva, H Fölster. 1996. Nutrient store and export rates of Eucalyptus urograndis plantations in eastern Amazonia (Jari). Forest Ecology and Management 80(1): 225-234.

Soil Survey Staff. 2010. Keys to Soil Taxonomy. 10th ed. Washington DC, USA. USDA-Natural Resources Conservation Service. 332 p.

Vaz de Arruda Silveira RL, EM Higashi. 2002. Nutriçao e adubuçao de Eucalyptus: viveiro e campo. In 1er. Curso de Capacitaçao em nutriçao mineral e adubaçao de Eucalyptus: viveiro e campo. Piracicaba, Brasil. 22-24 de maio de 2002.

Verkaik E, AG Jongkind, F Berendse. 2006. Short-term and longterm effects of tannins on nitrogen mineralisation and litter decomposition in kauri (Agathis australis (D. Don) Lindl.) forests. Plant and Soil 287(1-2): 337-345.

Wise PK, MG Pitman. 1981. Nutrient removal and replacement associated with short-rotation eucalypt plantations. Australian Forestry 44(3): 142-152. 